\title{
Tuberculous Spondylitis: Clinical Features of 36 Patients
}

\section{Halil Yanardag1, Cuneyt Tetikkurt2², Muammer Bilir', Sabriye Demirci'1, Bulent Canbaz ${ }^{3}$, Mucahit Ozyazar ${ }^{1}$}

${ }^{1}$ Department of Internal Medicine, Cerrahpasa Medical Faculty, Istanbul University, Istanbul, Turkey

${ }^{2}$ Department of Pulmonary Medicine, Cerrahpasa Medical Faculty, Istanbul University, Istanbul, Turkey

${ }^{3}$ Department of Neurosurgery, Cerrahpasa Medical Faculty, Istanbul University, Istanbul, Turkey

Email: ^tetikkurt@gmail.com, *tetikkurt@superonline.com

How to cite this paper: Yanardag, H., Tetikkurt, C., Bilir, M., Demirci, S., Canbaz, B. and Ozyazar, M. (2016) Tuberculous Spondylitis: Clinical Features of 36 Patients. Case Reports in Clinical Medicine, 5, 411-417. http://dx.doi.org/10.4236/crcm.2016.510057

Received: August 22, 2016

Accepted: October 25, 2016

Published: October 28, 2016

Copyright $\odot 2016$ by authors and Scientific Research Publishing Inc. This work is licensed under the Creative Commons Attribution International License (CC BY 4.0).

http://creativecommons.org/licenses/by/4.0/

\begin{abstract}
We report 36 cases of spinal tuberculosis who were evaluated at the Internal Medicine Department of Istanbul University between January 1990 and March 2016. Twelve cases were accompanied by active pulmonary tuberculosis while ten patients had a previous history of tuberculosis. Eight patients had diabetes mellitus; six patients were on chronic steroid treatment with a mean dose of $24 \mathrm{mg} /$ day while two patients used azathioprine and methotrexate. The dominant symptom was back pain that was present in $64 \%$ of the subjects followed by low grade fever (42\%), and malaise (38\%). Lytic and destructive lesions were noted in various vertebrae in all of the cases while four patients had spinal compression, and two patients had iliopsoas abscess. The preliminary diagnosis was myeloproliferative disease with vertebral metastasis in eight patients. Tuberculin test was over $15 \mathrm{~mm}$ in 20 patients (58.8\%) while ERS and Creactive protein were highly elevated in $78 \%$, and $84 \%$ of the patients. Diagnosis of tuberculosis was confirmed by culture of aspirated material from intervertebral disc space, collection under CT guidance, sputum or bronchial lavage, and by retrospective anti-tuberculous treatment response. MRI revealed compatible findings with spinal tuberculosis in $30(80 \%)$ patients. In endemic countries, tuberculous spondilitis should be considered in immunosuppressed patients with back pain. Current or past tuberculosis infection is not a reliable indicator for Pott's disease in these patients. Laboratory findings were not usually useful to support the diagnosis. The low sensitivity of the tuberculin test may lead to an erronous diagnosis. Sensitivity of vertebral radiography and CT was low. MRI was the most useful radiologic investigation for the diagnosis of spinal tuberculosis. Tuberculosis of the spine is a diagnostic impasse notably in immunodeficient patients for the clinician.
\end{abstract}

\section{Keywords}

Tuberculosis, Spinal, Vertebrae, M. Tuberculosis, Pott's Disease 


\section{Introduction}

Pott's disease, also known as tuberculous spondylitis, is one of the oldest demonstrated diseases of humankind. Bone and soft-tissue tuberculosis accounts for approximately $10 \%-15 \%$ of the extrapulmonary tuberculosis cases and between $1 \%$ and $2 \%$ of the total cases. Tuberculous spondylitis is the most common form of musculoskeletal tuberculosis, comprising $40 \%-50 \%$ of all patients [1] [2] [3] [4]. Spinal tuberculosis may be a diagnostic and a therapeutic challenge for the clinician. Firstly, the symptoms and the laboratory findings are nonspecific that may lead to a significant delay in diagnosis. Secondly, neural involvement occurs in approximately half of the patients causing irreversible damage if not promptly and adequately treated [2] [5]. Immunosuppression may be a significant risk factor for tuberculosis. The mechanism for increased susceptibility to infection in these patients is the alteration of chemotactic, phagositic, and bactericidal activity of the polymorphonuclear leukocytes. However, the role of immunosuppression for the development and outcome of spinal tuberculosis has not been determined.

Spinal tuberculosis which occurs in approximately in one percent of the patients with tuberculosis is still associated with significant morbidity and mortality [1]. In this retrospective study, we evaluated the clinical and diagnostic features of thirty-six patients with spinal tuberculosis. Diagnostic difficulties encountered for the identification of spinal tuberculosis and effect of immunosuppression on the clinical aspects of spinal tuberculosis were also assessed.

\section{Patients and Methods}

Our study group consisted of 36 Caucasian patients who were diagnosed with spinal tuberculosis at the Internal Medicine Department of Cerrahpasa Medical Faculty between January 1990 and June 2016. Patient data regarding the clinical features, laboratory, radiologic, and therapeutic findings were obtained from files of the patients seen at the Internal Medicine and Neurosurgery Departments for thoracolumbar symptoms. All patients had complete blood count, serum biochemistry, urine analysis, ESR, CRP, tuberculine test, chest X-ray, ECG, computed tomography, and magnetic resonance imaging. Sputum culture was done in $20, \mathrm{FOB}$ with bronchial lavage culture was carried out in 26, and tissue culture was performed in 30 patients.

Patient characteristics are shown in Table 1. All patients received anti-tuberculous treatment. The patients were followed-up for at least a minimum of six years as out patients after treatment.

Table 1. Characteristics of the patients.

\begin{tabular}{lcc}
\hline & Absent n (\%) & Present n (\%) \\
\hline History of pulmonary tuberculosis & $26(72 \%)$ & $10(28 \%)$ \\
Active pulmonary tuberculosis & $24(66.6 \%)$ & $12(33.4 \%)$ \\
Tuberculosis vaccination & $4(11 \%)$ & $32(89 \%)$ \\
İmmunosuppression & $20(55.6 \%)$ & $22(44.4 \%)$ \\
Symptoms & $13(36 \%)$ & $23(64 \%)$ \\
\hline
\end{tabular}




\section{Results}

Twenty two of the patients (61.7\%) were male and the mean age was $46.2 \pm 14.8$ years. Four patients (11.4\%) had cervical, three (7\%) had both cervical and thoracal, eighteen (52.9\%) had thoracal, eight (24\%) had lumbar, and three (8\%) had sacral involvement. Twelve (33.3\%) subjects had a prior history of pulmonary tuberculosis. Eight patients (24\%) had diabetes mellitus. The mean duration of diabetes was $9.4 \pm 3.2$ years. Six (16\%) patients were using steroid treatment at the time of diagnosis for an average of 4 months. Two (5\%) patients used azathioprine and methotrexate. The mean steroid dose was 24.8 $\pm 14.6 \mathrm{mg}$ methylprednisolone (minimum dose: $10 \mathrm{mg}$ and maximum dose $32 \mathrm{mg}$ ) per day. A total of 16 patients were under immunosuppression during the sutudy. The most frequent (64\%) symptom was back pain. Fever was present in $42 \%$, malaise in $38 \%$, night sweats in $28 \%$, weight loss in $24 \%$ of the patients while $36 \%$ were asymptomatic.

Mean ERS and CRP were $86.2 \pm 24.8 \mathrm{~mm} / \mathrm{h}$ and $72.4 \pm 20.6 \mathrm{mg} / \mathrm{L}$. The mean tuberculin induration was $14.6 \pm 5.4 \mathrm{~mm}$ while it was negative in $16(44.4 \%)$ patients. Radiologic evidence of current lung tuberculosis was observed in 12 (33.3\%) patients while fibrotic or calcific parenchymal radiologic lesions associated with previous infection were detected in 10 (27.7\%) subjects. Plain radiography revealed features of spinal tuberculosis in $14(38.8 \%)$ patients. Sputum culture was positive only in one patient while bronchial lavage culture grew $\mathrm{m}$. tuberculosis in 8 (22.2\%) patients. MRI was the most useful diagnostic tool that revealed pathologic findings of spinal tuberculosis in $72.2 \%$ and CT showed diagnostic lesions in $33.3 \%$ of the patients.

Two patients (5.8\%) had iliopsoas abcess. Computed tomography (CT) guided needle aspiration biopsy was performed in 30 patients $(83.3 \%)$ and 9 of these $(30.0 \%)$ had a histopathological diagnosis while $21(70.0 \%)$ were culture positive. Smear positivity of the needle aspiration material for acid-fast bacilli was obtained in $32 \%$ of cases while culture positivity was present in $72 \%$ of our cases. Histologic studies confirmed the diagnosis of spinal tuberculosis in $64 \%$ of the patients. The most common cytological finding was epithelioid cell granulomas observed in $82 \%$ of the patients. Lymphocytic infiltration was noted in $78 \%$ and granular necrotic background was present in $70 \%$, and scattered multinucleated and Langhans' giant cells were seen in $62 \%$ of the cases. CT guided needle aspiration biopsy provided the final diagnosis in $86 \%$ of the patients when both microbiology and pathology results were assessed together. In the remaining 8 (22.2\%) patients with clinically and radiologically suspected tuberculosis, the diagnosis was confirmed with response to antituberculous treatment. MRI was performed in $30(83.3 \%)$ patients and $80 \%$ of these cases had compatible MRI findings with vertebral tuberculosis.

All patients received antituberculous treatment with at least four drugs for a minimum of twelve months. The antitubeculous treatment regimen consisted of SM 1.0 g/day, and PRZ $20 \mathrm{mg} / \mathrm{kg}$ for two months with INH $5 \mathrm{mg} / \mathrm{kg}$, RIF $10 \mathrm{mg} / \mathrm{kg}$, and ETM 25 $\mathrm{mg} / \mathrm{kg}$. The mean duration of treatment was between 12 and 18 months. The clinical and laboratory features of the patients are shown in Table 2. Two patients were immobilized for two months and received concomitant rehabilitation therapy. All patients improved without a neurological deficit or complication. 
Table 2. Clinical features of the patients.

\begin{tabular}{lcc}
\hline & Absent $\mathrm{n}(\%)$ & Present $\mathrm{n}(\%)$ \\
\hline Positive tuberculine test & $16(44.4 \%)$ & $20(55.6 \%)$ \\
Radiologic features of previous pulmonary tuberculosis & $26(72.2 \%)$ & $10(27.8 \%)$ \\
Radiologic features of current pulmonary tuberculosis & $25(69.4 \%)$ & $11(30.6 \%)$ \\
Positive vertebral radiography findings & $24(66.6 \%)$ & $12(33.4 \%)$ \\
Positive spinal CT findings & $14(38.8 \%)$ & $22(61.2 \%)$ \\
Positive spinal MR findings & $6(20 \%)$ & $20(80.0 \%)$ \\
Sputum culture $(+)^{*}$ & $18(90.0 \%)$ & $2(10.0 \%)$ \\
Bronchial lavage culture $(+)^{* *}$ & $22(84.6 \%)$ & $4(15.4 \%)$ \\
Tissue culture $(+)^{* * *}$ & $9(30.0 \%)$ & $21(70.0 \%)$ \\
Tissue histopathology $(+)^{* * *}$ & $10(33.3 \%)$ & $20(66.7 \%)$ \\
\hline
\end{tabular}

Performed in: ${ }^{\star} 20$ patients, ${ }^{* * 26}$ patients, ${ }^{* * 30}$ patients.

\section{Discussion}

Tuberculosis of the spine is an uncommon form tuberculosis occuring in fewer than $1 \%$ of the patients with tuberculosis. Systemic symptoms and clinical findings usually arise late in the course of infection after significant spinal destruction has occured [1] [2] [3] [4]. Therefore, the diagnosis is a challenge for the clinician. In our study, we found that neither patient history, nor physical examination, or the laboratory findings were specific and sensitive for the diagnosis. We have observed that only the radiologic findings pointed out to the final diagnosis. But on the other hand, the presence of similar radiologic findings in malignant, metastatic, or fungal spinal involvement was the vulnerable point for plain radiography and CT. MRI examination was the most effective imaging study for spinal tuberculosis. The final diagnosis was only confirmed by the isolation of acid-fast-bacilli (AFB) in smear or culture from the lesion site.

Constitutional symptoms fever, weight loss, and night sweats were present in approximately $40 \%$ of the cases. Local back pain was the earliest and the most common symptom of spinal tuberculosis. Back pain occurred in $64 \%$ of the patients. Chronic back pain as the only symptom is observed in $61 \%$ of cases of spinal tuberculosis while the patient symptom profile was compatible with the findings of Pertuiset, Le Page, and Cormican et al. [3] [6] [7] [8]. Symptoms or history were not reliable or helpful for the immediate diagnosis of spinal tuberculosis in our study. Systemic symptoms and back pain were usually manifestations of advanced disease. Lumbar pain revealed mechanical destruction. Diagnosis of spinal involvement was not suspected in patients without tuberculosis history, evidence of radiologic findings of previous, or current pulmonary tuberculosis.

Erythrocyte sedimentation rate (ESR) is generally raised many folds and may be markedly elevated over $100 \mathrm{~mm} / \mathrm{hr}$ in the majority of patients with spinal tuberculosis [1] [3] [7]. Of the laboratory findings ERS and CRP were the most useful parameters in our study showing current inflammation. Although ERS and CRP were helpful, they were not sensitive or specific enough for accurate diagnosis. Tuberculine test results are 
positive in $84 \%$ - 95\% of patients with Pott's disease who are not infected with HIV [3] [6] [7]. PPD was only positive in $55.6 \%$ of our patients. Tuberculin test was not highly useful because its diagnostic accuracy was low in our patient group which is probably related to the high incidence of immunodeficient patients. The laboratory results were not reliable for pointing out to spinal tuberculosis.

The progression of spinal tuberculosis is usually slow and insidious with an average disease duration ranging from 4 to 11 months. Patients are admitted only when there is severe pain, marked deformity, or neurological symptoms [9] [10] [11]. In these patients, vertebral radiography still remains the cornerstone of spinal imaging. It often provides enough information for diagnosis and treatment of spinal tuberculosis. The plain radiograph may describe changes consistent with spine tuberculosis in up to $99 \%$ of the cases. The characteristic radiographic findings include rarefaction of the vertebral end plates, loss of disk height, osseous destruction, new-bone formation and softtissue abscess [9] [12] [13] [14]. Plain radiography and CT scan revealed destructive or inflammatory changes that occured late in the course of the disease. The main disadvantage of plain radiograph or CT was that they generally were normal in the early stages of the disease and only revealed manifestations of advanced spinal tuberculosis. Vertebral radiography with CT still remained the cornerstone of spinal imaging but it was not neither specific nor sensitive for early diagnosis. Using different pulse sequences, MRI revealed a better differentiation for soft tissue inflammatory and destructive lesions. MRI was efficient for demonstrating the features of spinal tuberculosis and appeared to be the most crucial step for the assessment of spinal tuberculosis that lead the clinician to final diagnosis. MRI was the most effective imaging study for demonstrating spinal tuberculosis. Chest X-ray and lung CT revealed revealed fibrotic or calcific lesions of pulmonary tuberculosis in a minority of patients. Current lesions of active pulmonary tuberculosis were also rare in our patients.

Spinal tuberculosis can present with atypical features resembling neoplastic or infectious lesions that necessitate the exclusion of such etiologies. The golden standart was CT guided needle aspiration biopsy which provided the final diagnosis in $86 \%$ of the patients. Smear positivity for acid-fast bacilli was low (32\%) while culture positivity was obtained in $70 \%$ of our patients. Positive smear and culture results were lower compared to other studies [7] [9] [11] [12] [13] [15]. Histologic studies confirmed the diagnosis in approximately two thirds of our patients. As with respiratory tuberculosis, culture may not be the gold standard for diagnosing spinal tuberculosis because mycobacterial bacilli may not be readily detected from lung or extrapulmonary sites. Therefore, diagnosis of spinal tuberculosis must be made on ground of clinical manifestations and radiology when bacteriology and pathology are negative or equivocal.

Because microbiologic studies may remain negative, histopathologic examination can be significant. In smear and culture negative patients, presence of granulomatous inflammation and caseification necrosis in the biopsy specimes was compatible with the final diagnosis which was confirmed with a positive antituberculous treatment response, retrospectively. Gross pathologic findings including exudative granulation tissue with interspersed abscesses was crucial for diagnosis. Coalescence of abscesses resulting in 
areas of caseating necrosis strongly supported the diagnosis of tuberculosis. Histopathologic examination may be the final step in the diagnostic pathway of spinal tuberculosis cases even it is not diagnostic on its own. In patients with compatible pathologic findings, retrospective evaluation of antituberculous treatment response after neoplastic and other infectious etiologies have been excluded, may be the best diagnostic approach in equivocal cases of spinal tuberculosis.

The small sample size warrants a more comprehensive study to confirm these findings. Further research with more heterogenous patient features is needed to describe the clinical findings of spinal tuberculosis. The second limitation is the presence of advanced disease with fibrotic tissue changes, thereby obscuring a positive culture result in such patients. The high ratio of immunosuppressed patients may be considered as the third limitation because the defective immune system may have a negative role on patient symptoms, tuberculin test results, and even on radiologic findings thereby beclouding or delaying the final diagnosis. The fourth limitation may be the inadequacy for discriminating acute and chronic cases since the diagnosis is much more difficult clinically for chronic cases.

\section{Conclusion}

Spinal tuberculosis is a major diagnostic challenge for the clinican. Although history and current symptoms are important, they are not reliable for diagnosis, especially in the immunodeficient patients. Tuberculine test is not useful because of the high incidence of false negative results that may lead to erroneous or delayed diagnostic outcomes in states of immunosuppression. Other laboratory results are neither specific nor sensitive for diagnosis and only may indicate current inflammation. Chest X-ray or CT may reveal pathologic findings of old tuberculosis infection that may lead to diagnosis but they are equivocal for final diagnosis. Plain vertebral radiography and CT may be helpful as the initial non-invasive diagnostic work-up of patients but their sensitivity is low. MRI is the most efficient diagnostic tool for demonstrating the features of spinal tuberculosis and appears to be the most crucial step for the assessment of spinal tuberculosis that may strongly indicate the final diagnosis. Clinicians should bear in mind that spinal tuberculosis can present with atypical features resembling neoplastic or infectious lesions that necessitate the exclusion of such etiologies. The patients may present with symptoms that do not point out to spinal disease or may be totally asymptomatic. Pott's disease appears as a diagnostic dilemma especially in immunodeficient patients since neither the patient history, the symptoms, nor the laboratory findings are sensitive or specific.

\section{Conflict of Interests}

There are no conflicts of interest to declare.

\section{References}

[1] te Beek, L.A., van der Werf, M.J., Richter, C., et al. (2006) Extrapulmonary Tuberculosis by 
Nationality, The Netherlands, 1993-2001. Emerging Infectious Diseases, 12, 1375-1382. http://dx.doi.org/10.3201/eid1209.050553

[2] Cheung, W.Y. and Luk, K.D. (2012) Clinical and Radiological Outcomes after Conservative Treatment of TB Spondylitis: Is the 15 Years' Follow-Up in the MRC Study Long Enough? European Spine Journal, 22, 594-602. http://dx.doi.org/10.1007/s00586-012-2332-x

[3] Le Page, L., Feydy, A., Rillardon, L., et al. (2006) Spinal Tuberculosis: A Longitudinal Study with Clinical, Laboratory, and Imaging Outcomes. Seminars in Arthritis and Rheumatism, 36, 124-129. http://dx.doi.org/10.1016/j.semarthrit.2006.04.007

[4] Leibert, E. and Haralambou, G. (2004) Tuberculosis. In: Rom, W.N. and Garay, S., Eds., Spinal Tuberculosis, Lippincott, Williams and Wilkins, 565-577.

[5] Turgut, M. (2001) Spinal Tuberculosis (Pott's Disease): Its Clinical Presentation, Surgical Management, and Outcome. A Survey Study on 694 Patients. Neurosurgical Review, 24, 8-13. http://dx.doi.org/10.1007/PL00011973

[6] Pertuiset, E., Beaudreuil, J., Liote, F., et al. (1999) Spinal Tuberculosis in Adults. A Study of 103 Cases in a Developed Country, 1980-1994. Medicine (Baltimore), 78, 309-320. http://dx.doi.org/10.1097/00005792-199909000-00003

[7] Cormican, L., Hammal, R., Messenger, J., et al. (2006) Current Difficulties in the Diagnosis and Management of Spinal Tuberculosis. Postgraduate Medical Journal, 82, 46-51. http://dx.doi.org/10.1136/pgmj.2005.032862

[8] Hsu, L.C., Cheng, C.L. and Leong, J.C. (1988) Pott's Paraplegia of Late Onset: The Cause of Compression and Results after Anterior Decompression. Journal of Bone \& Joint Surgery British Volume, 70, 534-538.

[9] Pertuiset, E., Beaudreuil, J., Lioté, F., Horusitzky, A., Kemiche, F., Richette, P., et al. (1999) Spinal Tuberculosis in Adults. A Study of 103 Cases in a Developed Country, 1980-1994. Medicine (Baltimore), 78, 309-320. http://dx.doi.org/10.1097/00005792-199909000-00003

[10] Skaf, G.S., Kanafani, Z.A., Araj, G.F. and Kanj, S.S. (2010) Non-Pyogenic Infections of the Spine. International Journal of Antimicrobial Agents, 36, 99-105.

http://dx.doi.org/10.1016/j.ijantimicag.2010.03.023

[11] Mondal, A. (1994) Cytological Diagnosis of Vertebral Tuberculosis with Fine Needle Aspiration Biopsy. Journal of Bone \& Joint Surgery American Volume, 76, 181-184.

[12] Polley, P. and Dunn, R. (2009) Noncontiguous Spinal Tuberculosis: Incidence and Management. European Spine Journal, 18, 1096-1101. http://dx.doi.org/10.1007/s00586-009-0966-0

[13] Azzam, N.I. and Tammawy, M. (1988) Tuberculous Spondylitis in Adults: Diagnosis and Treatment. British Journal of Neurosurgery, 2, 85-91. http://dx.doi.org/10.3109/02688698808999663

[14] Jain, R., Sawhney, S. and Berry, M. (1993) Computed Tomography of Tuberculosis: Patterns of Bone Destruction. Clinical Radiology, 47, 196-199. http://dx.doi.org/10.1016/S0009-9260(05)81162-6

[15] Shi, T., Zhang, Z., Dai, F., Zhou, Q., He, Q., Luo, F., Hou, T. and Xu, J. (2016) Retrospective Study of 967 Patients with Spinal Tuberculosis. Orthopedics, 39, e838-e343. http://dx.doi.org/10.3928/01477447-20160509-03 